\title{
La misère de position automobile : socialisation de classes et rapport social à l'automobile
}

The misery of automobile position: class socialization and social relationship to the automobile

Joseph Cacciari et Leslie Belton Chevallier

\section{(2) OpenEdition}

\section{Journals}

Édition électronique

URL : https://journals.openedition.org/popvuln/1314

DOI : 10.4000/popvuln.1314

ISSN : 2650-7684

Éditeur

LIR3S - Laboratoire Interdisciplinaire de Recherche "Sociétés Sensibilités Soin" (UMR 7366 CNRS-uB)

Édition imprimée

Date de publication : 1 décembre 2020

Pagination : 167-190

ISBN : 978-2-918173-28-1

ISSN : 2269-0182

Référence électronique

Joseph Cacciari et Leslie Belton Chevallier, «La misère de position automobile : socialisation de classes et rapport social à l'automobile », Populations vulnérables [En ligne], 6 | 2020, mis en ligne le 01 décembre 2021, consulté le 26 février 2022. URL : http://journals.openedition.org/popvuln/1314 ; DOI : https://doi.org/10.4000/popvuln.1314

Ce document a été généré automatiquement le 26 février 2022.

Les contenus de la revue Populations vulnérables sont mis à disposition selon les termes de la Licence Creative Commons Attribution 4.0 International 


\section{La misère de position automobile : socialisation de classes et rapport social à l'automobile}

The misery of automobile position: class socialization and social relationship to the automobile

Joseph Cacciari et Leslie Belton Chevallier

\section{Introduction}

1 Les politiques municipales de mobilité tentent aujourd'hui de réduire la place de l'automobile au cœur des villes pour diminuer les pollutions qui y sont associées: réduction des voies de circulation ou du nombre de places de stationnement, augmentation des frais de parking, diminution de la vitesse de circulation autorisée en ville, interdictions de circulation pour les véhicules les plus polluants, péages urbains, etc. Plusieurs travaux (Brown, 2017 ; Clark et al. 2016 ; Coquard, 2018; Démoli, 2015) pointent les conséquences inégalitaires de ces politiques de restriction de la voiture en ville vues comme une forme d'incitation "par le haut" à abandonner ou réduire l'usage de la voiture particulière. Ces auteurs montrent que la vie sans voiture (particulière) ne serait possible, à l'heure actuelle, que pour une petite fraction de ménages: des ménages urbains occupant des emplois suffisamment rémunérateurs, avec une maîtrise de leur agenda et un accès à l'emploi facilité dans des zones bien quadrillées par des offres de transports alternatives à la voiture individuelle en propriété.

2 Par leurs lieux de résidence ou d'emplois plus éloignés du centre ou moins bien desservis et le poids de l'automobile dans la structure de leurs dépenses (Nicolas et al., 2012), les classes populaires seraient plus vulnérables face à des mesures qui renchériraient leurs dépenses de déplacements. Au-delà du risque de tomber dans une trappe à pauvreté, les ménages les moins aisés se verraient contraints de renoncer à la voiture, un outil d'accès à l'emploi (Le Breton, 2005; Orfeuil, 2004) et un moyen de 
positionnement social (Coulangeon et Petev, 2012; Demoli et Lannoy, 2019). Les ménages les plus pauvres sont déjà plus immobiles que les autres ménages (MotteBaumvol et Belton Chevallier, 2019) mais sont aussi plus à même d'être "privés de voiture » (Mattioli et Colleoni, 2016). Les ménages populaires sont donc plus susceptibles de vivre les incitations institutionnelles à renoncer à la voiture (augmentation du coût d'usage, discours disqualifiant l'automobile) comme un facteur de vulnérabilisation de leurs situations par leurs mobilités quotidiennes. Celles-ci ont depuis longtemps été orientées par un usage intensif ou prioritaire de l'automobile. Ils peuvent y voir une stigmatisation de leur mode de vie quand il est fortement associé à ce mode de déplacement (Cacciari et Belton Chevallier, 2020).

3 C'est sur cette frustration relative que le mouvement des Gilets jaunes en France s'est d'ailleurs amorcé. Avant ce mouvement social, nombre d'enquêtes avaient déjà cherché à comprendre les ressorts de la mobilité des plus pauvres (Fol, 2009) : leur taux de possession automobile (Grimal, 2012), leur propension à alterner des périodes avec ou sans voiture (Klein et Smart, 2017) ou à acquérir plutôt des véhicules d'occasion peu sûrs qui en font les principales victimes d'accidents de la route (Grossetête, 2012). Pourtant, peu est dit sur la manière dont la disqualification relative de l'automobile comme mode de déplacement quotidien affecte la perception de l'identité sociale des personnes qui ne peuvent, pour une raison ou une autre, s'en passer. De la même manière, comment la voiture particulière peut-elle devenir un élément entraînant un sentiment de déclassement ou de vulnérabilisation sociale ? Le constat est valable pour tous les ménages, y compris les plus aisés. Comment les ménages en général vivent-ils le fait de devoir plus ou moins explicitement abandonner leur voiture? Plus généralement, si les liens entre les coûts objectivables de la voiture et son renoncement sont étudiés, quelle perception relative et relationnelle en ont les ménages? En quoi le fait de ne plus avoir de voiture est-il un signe ou stigmate de pauvreté ? Malgré l'implacable domination quantitative de la norme automobile pour les mobilités quotidiennes et de la diffusion massive du permis de conduire en France, il y a beaucoup à apprendre sur la variété sociale des expériences vécues de cette culture, y compris dans le sens où elle exercerait son influence inégalement selon la classe, le genre, la génération (Démoli et Lannoy, 2019).

L'objectif de cet article est de saisir dans quelles mesures la voiture et son abandon peuvent être vécus comme une forme de vulnérabilisation d'une situation socioéconomique, une menace sur le style de vie ou un appauvrissement. Au-delà des considérations bien documentées sur la misère absolue de certains ménages par rapport à l'automobile, qui peinent à suivre les circuits officiels d'achat, de réparation ou d'approvisionnement en carburant (Collectif Rosa Bonheur, 2019 ; Démoli, 2017), cet article souhaite saisir en quoi la voiture, ses caractéristiques ou son abandon peuvent être associés dans les perceptions à une pauvreté plus relative ou relationnelle.

5 Si le niveau absolu de ressources économiques à la disposition des individus peut expliquer leurs équipement et usages automobiles, ces mêmes variables peuvent être examinées comme des modalités de représentations subjectives de la position occupée dans le monde social, comme le suggèrent plusieurs travaux cités ici. Ces représentations ne sont cependant pas à comprendre au sens usuel de la psychologie individualiste. Dans notre enquête, et après d'autres citées ci-dessus, ces prises de position à travers les formes d'usage et d'équipement automobile sont apparues comme 
relationnelles, car construites dans des rapports à la fois antagonistes et contradictoires avec la position d'autres personnes ou groupes.

6 En partant de cette idée, notre propos est d'examiner les figures et ressorts d'une éventuelle misère de position automobile, directement inspirée des travaux de Pierre Bourdieu et de son équipe dans La Misère du monde (1993). La misère de condition (économique et sociale) renvoie à une pauvreté absolue, celle des ressources d'existence et de l'absence d'horizon de sortie de cet état et engendre de nombreuses fragilités pour accéder à des ressources (économiques, culturelles, etc.), à la possession d'un logement ou d'une voiture. La misère de position est liée à l'hétéronomie sociale, économique et normative à laquelle sont subordonnées les personnes et qui les amène à se percevoir comme pauvres par rapport à d'autres personnes ou groupes sociaux. Nous optons pour cette approche par les rapports sociaux et les différences de positions sociales dans la mesure où la voiture, son usage autant que sa possession, renvoient au soi socialisé et à son appréhension dynamique. En effet, tel ou tel type de rapport à la voiture (usage et possession) n'existe qu'au sein d'un espace de positions différentielles, interdépendantes, antagoniques et contradictoires au sein duquel la mobilité automobile fait partie du monde social et des autres dimensions constitutives des styles de vie (comme le rapport au travail et à l'emploi, les styles de consommation, les (dé)goûts culturels, etc.).

\section{II. Éléments méthodologiques}

7 Pour explorer les rapports sociaux à l'automobile, nous nous appuierons sur les résultats d'une enquête par entretiens de 60 à 210 minutes réalisés dans quatre aires urbaines françaises (Bordeaux, Dijon, Lyon et Paris) en 2018, avant les Gilets jaunes, et dont la composition est précisée dans le tableau 1. Si certains profils sont plus fréquents (cinquantenaires, femmes, vivant dans l'urbain et issus des classes populaires) que d'autres, la diversité des trajectoires (spatiales et sociales) est satisfaisante. Les cinquante et une personnes enquêtées ont été repérées comme démotorisées via le panel Parc Auto (TNS Kantar) qui décrit de manière annuelle l'équipement automobile des Français. Parmi ces ménages, certains avaient renoncé totalement à la voiture, d'autres y ont renoncé partiellement et possèdent encore des véhicules.

Les entretiens ont permis d'explorer les trajectoires biographiques d'individus du point de vue de la voiture en général, de leurs équipements automobiles en particulier ou encore des savoirs et savoir-faire, manières de penser l'usage de ce mode ou d'autres. Ces entretiens ont été l'occasion de lier plusieurs dimensions des trajectoires: résidentielles, familiales, professionnelles, amicales ou autres. À travers ces biographies, nous avons étudié les mécanismes de (re)configuration du rapport à l'automobile. Au-delà des ressorts de la démotorisation, l'article veut surtout saisir différentes situations du rapport social à l'automobile et à son relatif abandon. Ce renoncement est encore examiné de manière quantitative sous l'angle d'une vulnérabilité physique ou économique, en ciblant des ménages très pauvres (Demoli, 2015 ; Froud et al., 2002) ou des personnes âgées en situation de dépendance (Espinasse, 2005). Pourtant, ces ménages démotorisés sous contrainte sont à distinguer de ménages démotorisés et mobiles comme ceux des grands centres urbains avec un style de vie en rupture avec la motorisation (Demoli, 2017). Nous voulons ici explorer différentes 
figures d'un rapport social spécifique à l'automobile : la misère de position automobile, c'est-à-dire celle qui dépend de conditions économiques, mais aussi d'une pression normative hétéronome en termes de mobilité spatiale.

9 Trois figures de misères de position peuvent être identifiées : une acceptée sous le registre du fatalisme, une redoutée dans la crainte d'un déclassement au quotidien et une transfigurée. Dans cette dernière, les individus concernés conservent des ressources matérielles et symboliques les aidant à ériger leur misère de position automobile en composante de leur style de vie, et ce bien que leur rapport à l'automobile les déclasse sur d'autres aspects. Dans le vocable bourdieusien, il s'agirait d'une bonne volonté de démotorisation.

Tableau 1. Principales caractéristiques de la population enquêtée (en effectif)

\begin{tabular}{|c|c|}
\hline Caractéristiques & Effectifs \\
\hline \multicolumn{2}{|l|}{ Sexe } \\
\hline Femme & 29 \\
\hline Homme & 22 \\
\hline \multicolumn{2}{|l|}{ Âge } \\
\hline Moins de 40 ans & 8 \\
\hline Entre 41 et 60 ans & 22 \\
\hline 61 ans et plus & 21 \\
\hline Moyenne & 58,2 ans \\
\hline Médiane & 57,5 ans \\
\hline \multicolumn{2}{|l|}{ Type de logement } \\
\hline Appartement & 40 \\
\hline Maison & 11 \\
\hline \multicolumn{2}{|c|}{ Statut d'occupation } \\
\hline Hébergé.e & 2 \\
\hline Location privée & 6 \\
\hline Location sociale & 9 \\
\hline Propriétaire & 34 \\
\hline \multicolumn{2}{|c|}{ Statut professionnel d'Ego } \\
\hline Actif.ve occupé.e & 26 \\
\hline
\end{tabular}




\begin{tabular}{|l|l|}
\hline Inactif & 2 \\
\hline Retraité & 19 \\
\hline Chômage & 4 \\
\hline Statut matrimonial \\
\hline En couple & 15 \\
\hline Veu.f.ve & 5 \\
\hline Célibataire & 9 \\
\hline Divorcé.e, séparé.e & 22 \\
\hline Enfant mineur & \\
\hline Oui & 12 \\
\hline Non & 39 \\
\hline Nombre de voiture(s) \\
\hline 0 & 26 \\
\hline 1 & 13 \\
\hline 2 ou plus & 39 \\
\hline
\end{tabular}

\section{L'automobile comme signal de la position sociale}

10 L'un des principaux résultats de notre recherche est que le rapport à l'automobile s'acquiert tout au long de la trajectoire biographique, et n'est certainement pas homogène d'un territoire ou d'une classe sociale à l'autre. Cette socialisation à une certaine forme de rapport à l'automobile tire parti de trois dimensions qui font configuration dans le parcours des individus. D'abord, elle vient des ressources personnelles, culturelles, relationnelles ou économiques, pouvant être aussi collectives et avoir trait à une appartenance territoriale. Avoir été socialisé à proximité de pistes cyclables sécurisées ou avoir vécu dans une commune mal desservie en transports en commun infère nécessairement la perception de l'automobile et de la position sociale des individus vis-à-vis de cet objet. La socialisation à l'automobile implique, ensuite, une pluralité d'instances socialisatrices : de la famille à l'école, du groupe de pairs à la socialisation par et au travail, etc. Les relations entre les ressources et les mécanismes de socialisation sont, enfin, médiatisées par des expériences concrètes impliquant des propriétés telles que le genre, la classe sociale ou l'appartenance ethnique donnant une tonalité subjective à la culture automobile des individus. Cette configuration de socialisation induit l'intériorisation d'un certain rapport aux usages et à la possession de l'automobile dans le temps, qui peut évoluer. Cette approche dynamique de la 
socialisation automobile est au cœur de l'analyse proposée afin de montrer comment peut advenir le sentiment d'une misère de position automobile au cours d'expériences vécues au quotidien en relation avec autrui.

Le cas de Benoît est révélateur de la variété des expériences vécues en matière de mobilité automobile et de l'intériorisation, à travers elles, d'une perception de sa propre position dans l'espace social au prisme des pratiques de mobilités spatiales. Benoît a 68 ans au moment de l'enquête, est marié, a deux enfants adultes et des petitsenfants. Ses premières années de vie ont lieu en Charente-Maritime, au cœur d'un quartier urbain calme et relativement bourgeois. Arrivé en région parisienne à l'adolescence, suite au décès de sa mère, il vit aujourd'hui dans une commune de Grande Couronne parisienne, où il a tenu un commerce avec sa femme, avant de partir à la retraite et après une première carrière dans la réparation agricole et le démarchage commercial. Les premières expériences automobiles de Benoît sont contrastées. Peu présente dans son enfance, ses souvenirs liés à l'automobile sont plutôt positifs et précis.

[...] peu de gens avaient des voitures, finalement à ce moment-là. Alors, mon cousin, lui, il avait une voiture. Il avait même des belles voitures, il avait... je me souviens de sa dernière voiture, c'était une Panhard Dyna $\mathrm{X}$. Ah, ça, c'était une belle voiture tout en aluminium. Et alors, les voitures, c'étaient des petites voitures populaires, des Simca Aronde, les Dauphines, le début des Renault 8.

Sa mère n'a pas tout de suite eu de voiture. Elle a cependant transmis à Benoît le goût du voyage, d'abord en train, puis avec les voitures perçues avant tout comme des moyens de déplacement.

Par contre, ma mère, c'est elle qui m'a donné le goût des voyages, et quand elle s'est retrouvée, donc, seule, quand mon grand-père a disparu, donc, elle s'est acheté une voiture. Et elle a voulu avoir la voiture dont elle avait été folle à une époque, donc, qui était juste avant-guerre, c'était la traction avant. Donc, elle s'est acheté une Traction avant... Qui était une voiture déjà désuète, à l'époque, puisque la DS était déjà là. La DS, c'est en [19]57, la 1ère DS. Et j'ai perdu mon grand-père, je devais avoir $7-8$ ans. Je suis né en [19]51, donc, ça devait être [19]58, par là... Donc, les DS étaient déjà sur le marché, donc, la Traction, on en voyait, mais ce n'était pas la voiture dernier cri... parce que la Traction, elle datait de [19]30... La 1ère Traction je crois que c'est [19]36 - [19]37, quelque chose comme ça. Mais ma mère avait été folle dingue de la Traction, et donc, elle s'est dit « je veux une Traction », donc, elle s'est payée une 11 légère et la 11 légère, c'était la plus belle des Tractions. Parce qu'il y a eu la 15 qui était une grosse Traction lourde... lourdingue. La 11, c'est une belle voiture. Et donc, quand elle a eu sa Traction, elle s'en est donné à cœur joie.

13 La socialisation au voyage en voiture a marqué les pratiques de Benoît en définissant un rapport où, comme il le dit, "un lieu ça se mérite ». Plus fondamentalement, il a intériorisé une relation ludique et pratique à l'automobile, moyen d'évasion et objet de passion. Pour Benoît, la voiture est aussi classée et classante. Elle est classée, car il associe des modèles de véhicules à des usages et à des catégories sociales. La voiture est aussi classante, car rouler dans tel ou tel type de voiture et en avoir tel ou tel usage sont autant de modalités qui font les usagers. En plus de l'exemple de la traction de sa mère, une illustration de la disposition au classement social par l'automobile se trouve dans l'évocation des pratiques automobiles de son beau-frère.

Et donc, mon beau-frère, quand ils se sont connus avec ma sœur, il avait une petite voiture de célibataire, une Simca 1000, à l'époque, et après, qu'est-ce qu'il a acheté ? Il a acheté une 403, comme son papa. Une 403 Peugeot, qui était déjà une voiture de bourgeois installé, alors qu'ils étaient jeunes, encore, eux... Ils avaient quoi ? Ma 
sœur, si elle avait 23, lui devait avoir, je ne sais pas... Quand il a eu sa 403, il devait avoir 27 - 28 ans, peut-être. Ça faisait pour avoir une voiture de bourgeois, comme ça. Enfin, c'était la voiture qu'il voulait et, coup de chance quand même, il aimait bien se balader en voiture, lui. Oui, c'est vrai qu'il aimait bien se balader, finalement, lui aussi, pas de la même manière que moi, j'entends la balade ou que l'entendait ma mère, mais il aimait bien faire son tour en voiture.

[Suite à une demande de précision sur ce point]

Alors, pas de la même manière. Ma mère aimait se balader pour la balade. Le mode de transport n'était pas une fin en soi, c'était un vecteur. Voilà, on prenait le train parce qu'on n'avait pas d'autre choix, après, on prenait la voiture parce qu'elle avait une voiture. Mon beau-frère, lui, c'était le plaisir de se balader en bagnole. »

14 Ces extraits d'entretien étayent l'idée d'une socialisation générant des savoirs et des savoir-faire en matière de rapport à l'automobile. Benoît perçoit modèles et usages depuis une expérience lui ayant enseigné la variété des profils de conducteurs et de conductrices. À travers les formes de possession et d'usages, il peut décoder les styles des unes et des autres. Il s'appuie sur des indices comme l'âge, le milieu social perçu et leurs expériences (la peur chez sa sœur, le sentiment du voyage chez lui ou sa mère, la balade pour la balade pour son beau-frère) pour expliquer des pratiques variées de l'automobile et amener à considérer telle marque et tel modèle comme désirables ou non. Benoît évoque ces dimensions depuis sa propre expérience, de sa prime enfance, de sa trajectoire d'adulte et de sa position sociale, au cours desquels ses méthodes de classement ont été éprouvées en interaction avec d'autres.

Le rapport à l'automobile de Benoît est dominé par un usage parcimonieux et utilitaire (pour les voyages) avec une inclinaison à la possession de véhicules modestes relayée par une pratique de collection de modèles prestigieux en version miniature. Il n'aurait pas contracté un crédit automobile pour s'acheter un modèle luxueux et il n'a possédé que des modèles d'entrée de gamme ou d'occasion. Benoît fait partie des dominés de ce que l'on pourrait appeler l'espace des positions de la mobilité automobile. Il accepte sa position en tant que telle du fait d'une socialisation ayant produit une inclinaison à la lucidité sur les limites objectives de ses possibilités matérielles.

Dans une autre génération, une autre classe sociale et depuis le genre féminin, un rapport à l'acceptation de la misère de position automobile se retrouve avec un caractère sensiblement différent. Retraitée depuis quelques mois après une carrière de conseillère en économie sociale et familiale, Paulette a 60 ans et vit en petite couronne parisienne avec deux de ses trois filles qui ont entre 25 et 32 ans. Paulette a grandi à la campagne en Champagne sans voiture et a été socialisée sur le tard à l'automobile, suite à son arrivée à Paris. S'il y a eu une misère de position automobile objective pour Paulette, celle-ci résulte d'une obligation à posséder et utiliser régulièrement un véhicule pour améliorer sa situation professionnelle qui consistait à accompagner et donc rendre visite à des familles en difficultés (sociales, financières, etc.) dans leur vie quotidienne. Paulette n'a pour ainsi dire pas eu le choix. Mais elle trouvait des raisons, subjectives, de l'accepter.

J'ai toujours considéré que c'était un choix que je faisais de confort, c'est ma maison, ma voiture. C'était mon chez-moi [...] Entre deux interventions, c'est le sas de décompression, et c'est le lieu où je pouvais me reposer, où je pouvais lire, où je pouvais téléphoner, où je pouvais manger.

Sous les contours de l'obligation à posséder une voiture, la situation d'acceptation de misère de position automobile n'est pas ici induite par une socialisation précoce à la voiture personnelle. Elle vient d'une familiarisation avec un état des choses où la 
voiture apparait comme la seule alternative crédible pour gagner en confort et en temps dans un métier d'exécutante qui l'obligeait à se déplacer. L'automobile constituait pour Paulette une composante de la reproduction de sa force de travail.

Aujourd'hui à la retraite et possédant encore une voiture personnelle, Paulette se considère comme démotorisée, car elle utilise peu sa voiture, lui préférant la marche. Elle conserve cette automobile par réactivation de sa disposition à la recherche de « confort",

pour accompagner ses filles à l'hôpital, pour aller voir sa sœur en région, ou lorsqu'il n'y a pas d'alternative crédible à la voiture selon sa perception :

Enfin, 50 km aller, donc, j'y vais là, en voiture. Ma sœur qui habite en Haute-Saône, aussi, je vais chez elle en voiture, parce que c'est pareil, la gare est à je ne sais pas combien de kilomètres de chez elle et j'aime bien être indépendante. Par contre, je vais dans les Côtes-d'Armor, j'ai des amis là-bas. Ils habitent Lamballe, je vais en train. À partir du moment où je suis toute seule et où c'est accessible en train ou en bus, parce que je prends les bus Macron, aussi, de temps en temps, voilà. J'ai une amie à Strasbourg et je jongle entre les billets. Maintenant où je suis en retraite. Surtout maintenant. Je jongle avec les billets de train moins chers et les bus.

Ces deux cas de misère d'une position dominée vis-à-vis de l'automobile relativement acceptée, voire occultée comme telle, montrent que celle-ci ne joue pas nécessairement comme un stigmate. Par ses caractéristiques (présence, usage, marque, taux de possession, état), la voiture est un indicateur pour Benoît et Paulette de la position occupée par les individus dans l'espace social. Mais elle est aussi un support de réflexivité sur cette position, ses contradictions, son origine.

\section{La vulnérabilisation par l'automobile ou la misère de position comme contrainte subjective}

20 Avec Benoît et Paulette, la voiture est apparue comme un objet classé et classant qui joue comme une modalité de positionnement social à travers des propriétés sociales tel que l'âge ou l'appartenance à une classe sociale, par exemple. C'est un résultat relativement connu des grandes enquêtes statistiques qui montrent bien les ressorts sociaux de la possession automobile (Coulangeon et Petev, 2012). Prolongeant ces travaux, notre enquête par entretiens fait ressortir la forme de rapport direct que ce classement social par l'automobile peut induire en termes de conséquences pratiques ou d'auto-positionnement par rapport à autrui. En admettant que la socialisation est une affaire de représentation mentale, de savoirs et savoir-faire pratiques, de positionnement en action, on peut dès lors comprendre comment la voiture entraîne, pour un ménage ou un individu, une inclination à se sentir vulnérable socialement et économiquement, à réexaminer son appartenance sociale. Cette conception va au-delà de la seule composante économique de la pratique de l'automobile. Elle montre que l'automobile, comme d'autres objets, peut venir matérialiser un écart entre deux états d'une situation sociale et économique.

Claire, 42 ans, vit à Paris depuis une dizaine d'années avec ses 4 enfants. Si elle est née en région parisienne, elle part vivre dans une région rurale et montagneuse à l'adolescence suite au divorce de ses parents. Le décès de son mari l'amène à choisir de revenir vivre à Paris pour se rapprocher de sa famille. Les extraits suivants font le récit de ce retour du point de vue de sa trajectoire automobile : 
[Sur le choix de garder une automobile malgré les difficultés économiques]

Ben l'habitude, c'est vrai que je ne sais pas... et puis j'avais quand même des enfants petits, et j'avoue que du coup, en m'en séparant, j'avais eu une grosse frayeur de ne pas y arriver. Parce que oui pour moi, famille avec enfants, voiture obligatoire. [...] Je m'étais dit que j'étais plus à l'essai... je pensais quand même que vraisemblablement dans les 6 mois qui allaient venir, j'allais devoir racheter une voiture. Et finalement non !!! Parce que c'est vrai finalement je me rends compte que de ne pas avoir ce stress de garer la voiture, des dégradations...

[Sur le coût de cette décision]

Voilà. Sans même voir l'aspect financier, ce n'est pas une motivation purement financière. [...] Parce que c'est vrai que quelque part être en possession d'une voiture à Paris, c'est un stress, et puis je sais que j'allais toujours, déjà, la regarer, parce que souvent je n'arrivais pas à me garer bien, j'étais obligée de la garer mal, tard le soir, de se lever à $7 \mathrm{~h}$ du matin pour aller la regarer... Il fallait payer quasiment tous les jours. Et puis du coup je sais qu'en revenant de l'école, on essayait de passer à côté de la voiture pour voir si elle ne s'était pas fait vandaliser trop. [...] Je pensais que ce serait un problème de ne pas avoir de voiture et finalement ça a été une libération.

L'usage quotidien de l'automobile dans sa précédente localisation résidentielle amène Claire à ne pas se séparer de son véhicule une fois revenue à Paris. Pour autant, elle finit par expérimenter les différents coûts liés à la possession d'une voiture dans un territoire très dense et où la place de l'automobile n'est pas la même que dans le monde rural, dans les faits et les discours. Au-delà du prix du stationnement, elle expérimente surtout le poids psychologique de devoir gérer le stationnement d'un véhicule qui bouge peu. Elle anticipe des dépenses (amendes, enlèvement, fourrière puis frais d'entretien importants dus au vieillissement de sa voiture) qui, mises en miroir avec son recours effectif, lui font craindre une dévalorisation de son capital et un appauvrissement si ces dépenses viennent à se concrétiser. L'appauvrissement par l'automobile est ici plus redouté ou anticipé que réel. Néanmoins ce sentiment se cumule à un autre ressenti, qui est celui d'un déclassement par l'automobile et plus précisément par la possession automobile. Quand elle vivait à la montagne avec ses enfants, posséder une automobile était considéré comme normal. Mais en s'installant à Paris, le raisonnement semble différent, surtout dans son cercle de sociabilité :

J'ai un certain nombre d'amis qui n'avaient pas de voiture, enfin, qui n'ont jamais eu de voiture, des purs parisiens qui n'ont jamais habité nulle part d'autre, qui du coup n'ont jamais eu de voiture, la majorité des gens d'ailleurs. Après il y a quand même des gens qui ont des facilités, des parkings et qui du coup conservent une voiture.

23 Pour sa part, entre des services de mobilité (elle est abonnée à un système de vélos en libre-service, à un service d'autopartage, à un programme fidélité d'une agence de location) et des transports collectifs à courte ou longue distance, Claire se considère plus en phase avec sa position sociale actuelle, au point de se qualifier de "folle" d'avoir gardé son véhicule aussi longtemps. Si Claire a redouté une perte d'autonomie par le renoncement automobile, elle a expérimenté temporairement cette situation par crainte d'un déclassement associé au fait de ne plus pouvoir assurer sa position de mère avec enfants à charge, position qu'elle associe à la nécessité d'avoir une voiture. Elle transfigure ensuite cette situation de contrainte par un ensemble de stratégies de mobilité.

24 Le cas de Géraldine, 52 ans, mariée et sans enfant, est différent car sa condition économique n'est pas déterminante dans ses choix automobiles. Géraldine semble $a$ 
priori peu concernée par la misère de condition automobile. Elle est issue d'un milieu bourgeois. Son mari et elle sont tous les deux cadres, ont des revenus importants et vivent au cœur de Paris. Socialisée tôt et de manière intensive à la voiture («tout le monde prenait la voiture pour tout»), elle dit avoir du mal à vivre son renoncement pourtant ancien à la voiture :

Nous, on est très adaptables, ça, ce n'est pas le problème. Non, et puis par contre, quand on est rentrés en France... Enfin, à Paris, les premiers temps, j'avais une voiture, et puis après, quand j'ai commencé à devoir mettre 1 heure, $1 \mathrm{~h} 30$ le samedi pour... à tourner pour pouvoir me garer. J'ai loué un garage... qui me coûtait une fortune. [...] et puis en plus, c'était quand même à un quart d'heure à pied, enfin...

[Sur la décision de revendre son véhicule]

Et puis après, j'en ai... j'ai fini par la vendre parce que ce n'était pas que chez moi où je mettais $1 \mathrm{~h} 30$, c'était partout, on ne pouvait plus se garer parce qu'il y avait des Vélib, parce ce qu'il y avait des... Parce qu'on supprimait les places de parking, madame Hidalgo supprimait les places de parking un peu partout. C'est contraint, que j'ai abandonné la voiture. [...]

[Sur les pratiques de mobilité après revente]

Ben, je l'ai revendue... 3-5 ans après [son retour]. Non, j'ai quand même essayé. Je suis entêtée. Mais maintenant, je fonctionne avec les véhicules de location, lorsque je suis en vacances... Transports en commun et véhicules de location, lorsque j'ai besoin d'une voiture. Ou taxi, taxi quand j'ai besoin de me déplacer dans Paris, enfin, taxi quand il n'y a pas de transports en commun, j'entends.

Géraldine aime conduire et regrette de ne plus pouvoir le faire. Elle tient cependant à rester dans l'hypercentre parisien pour profiter des aménités sociales et culturelles. Elle ne s'imagine pas vivre en banlieue ou en " province ». Elle est prise dans une forme de contradiction entre, d'un côté, des pratiques culturelles ou résidentielles qui lui conviennent et qui sont conformes à sa position dominante en termes de situation, et, de l'autre côté, un renoncement à la voiture qui traduit une position dominée au sein des classes dominantes et qui l'éloigne du cadre pourtant bourgeois et élitiste dans lequel elle a été socialisée.

La voiture a toute sa place dans cette appréciation contradictoire de sa situation. Ainsi, ses parents et ses frères et sœurs ont toujours changé régulièrement de voitures en prenant des modèles neufs. De fait, elle a eu peu de véhicules à son nom en partie du fait d'une carrière internationale en tant qu'expatriée en Afrique et au Moyen-Orient. Dans ce cadre, son mari et elle ne conduisaient pas mais disposaient d'une voiture avec chauffeur qui matérialise une forme de domination par l'automobile, par la pratique et moins par la possession. Si, à Paris, Géraldine continue de se faire parfois conduire par des taxis et si elle utilise de manière agile la marche ou les transports en commun, l'impossibilité objective de pouvoir posséder un véhicule vient la gêner par la dissonance sociale qu'elle induit entre sa condition (de non-automobiliste) et sa position (de classe), décalage largement construit dans différentes instances comme sa famille ou sa sphère professionnelle.

\section{Une misère de position automobile transfigurée}

Les deux exemples précédents montrent que les individus peuvent être dans la crainte d'être « appauvris » travers la reconfiguration des possibilités d'usages et de possession automobile. Il s'agit d'un processus qu'on peut qualifier de vulnérabilisation. En tant qu'objet symbolique 
attaché à des hiérarchies sociales, la voiture est perçue comme un reflet indirect de sa position dans l'espace social et géographique. Ainsi l'état d'une voiture, sa taille, sa couleur, sa marque, son kilométrage, l'aisance à la conduire ou sa possession tendraient à envoyer un signal sur la position sociale. Néanmoins, les perceptions en la matière des individus et donc les représentations de leur propre positionnement par l'automobile ne sont pas figées et peuvent évoluer.

Prenons les cas similaires de Christophe et de Ludovic. La quarantaine, ils vivent en couple et ont des enfants encore mineurs à charge. Ils résident tous deux en banlieue parisienne, l'un en petite couronne et l'autre en grande couronne. Ils ont pour point commun d'avoir grandi en dehors de la région parisienne, respectivement en Gironde et dans le Pas-de-Calais, au sein de familles de "petits moyens » (Cartier et al., 2008). Dans cette configuration, la voiture prend très tôt une place importante pour les déplacements quotidiens : «J'étais à la campagne, donc du coup, la voiture, c'est un peu obligatoire, sinon, c'est la défaite [le fait d'être dans une position compliquée] pour se déplacer » (Christophe).

Ludovic et Christophe ont réalisé leur apprentissage de la conduite par la méthode de la conduite accompagnée à 16 ans et ont passé leur permis le jour de leurs 18 ans. Respectivement artisan et secrétaire pour Christophe et ouvriers pour Ludovic, leurs parents considèrent le permis comme "un passage obligé de la vie d'adulte" (Ludovic) et le financent.Ils jouent aussi un rôle important dans la constitution du pécule nécessaire à l'achat du premier véhicule en aidant Christophe à alimenter son livret d'épargne ou en trouvant des emplois saisonniers à Ludovic dès ses 16 ans. Dans les deux cas, la voiture semble centrale dans le cercle familial, mais aussi amical de jeunes adultes, plus particulièrement pour Christophe :

J'ai changé pas mal de voiture, après, derrière, oui, ça m'est arrivé, oui.

[Sur les médias automobiles]

Oui, oui. Et puis, bon, j'avais des potes qui... qui aimaient ça aussi. Et plein de potes qui étaient dans la mécanique, donc...On regardait les émissions comme auto-moto. On avait plein de potes qui changeaient, aussi, régulièrement de bagnole, donc, du coup, c'était... voilà... On se montrait ça... [...] Et puis il y a des équipements, et puis... voilà. On était... oui, c'était très dans ça [les voitures].

Socialisés tôt et intensivement à l'automobile dans un contexte où son absence est synonyme d'incapacité à se déplacer, d'accéder à des emplois ou des formations universitaires, Ludovic et Christophe en viennent pourtant à changer radicalement de cadre de vie en étant mutés en région parisienne. S'ils racontent encore avec émotion leurs premières expériences en transports collectifs, l'abandon de la voiture et de son usage semble relever d'une logique guidée par un changement de perceptions de l'objet, de ses avantages et de ses inconvénients, mais aussi de son prestige :

Mais, assez vite... assez vite, j'ai arrêté, en fait [de prendre la voiture].

[Sur l'impact de cette décision dans l'entourage et le fait d'en avoir discuté]

Ben, un peu, je crois que ça s'est fait aussi un peu dans le cercle d'amis, où, au bout

d'un moment, qui me disent «il faut que tu arrêtes avec ta bagnole, ça n'a pas d'utilité ». C'est assez récent, oui, et en plus, j'ai eu ça comme discours, parce qu'on a... on a des potes aussi, qui sont très bagnole, encore, pour le coup, des vrais parisiens. Et ils se déplacent tout le temps en voiture. Et c'est assez drôle de voir l'effet un peu inverse des discussions, en disant, aujourd'hui, enfin... Tout est fait vraiment pour faciliter, on va dire, les déplacements, puis... voilà, on poursuit un peu mieux le sujet pécuniaire, ça devient très cher, et voilà, ça, c'est une évidence, aujourd'hui, on peut tout faire, sans voiture. [...] 
Mais, même quand je vais sur Bordeaux. Oui, je ne prends plus la voiture, c'est fini. Ah, j'en ai ras-le-bol. En fait, je crois que j'ai eu un effet de saturation, au bout d'un moment... Et... de voir... de voir comment on se fait chier, en fait, sur la route, il faut payer, il faut payer, on ne fait que payer, ça commence à me gonfler, et puis voilà, les emmerdes.

[Sur les ennuis associés]

Ben, il faut faire le contrôle technique, il faut réparer le truc, les machins... Mais ça

a provoqué... peut-être, chez moi, oui, c'est un dégoût. (Christophe)

\section{l'automobile pour Christophe et sa famille. Le basculement se fait d'une mobilité} polarisée par la voiture à une mobilité démotorisée au quotidien, où la voiture n'est plus un objet indispensable socialement, comme en témoignent Christophe et Ludovic ci-après.

Je ne me suis jamais dit «si je prenais la voiture, ce serait mieux». Dans les premières années où on était sur Paris ça nous est arrivé de prendre la

voiture

pour le loisir, en entrant sur Paris, et on l'a systématiquement très amèrement regretté. [...] Alors, les images que j'avais de la voiture dans Paris, c'était bloqué à l'Arc de Triomphe, à tourner pendant 3 jours. Donc, c'était l'image que j'avais, donc, oui, j'ai... même si c'était des préjugés parce que je n'étais pas venu avant, ni avec mes parents ni personne, c'était vraiment... c'était des préjugés, et donc, j'y étais, et j'ai été conforté tout de suite par les transports [...] (Christophe).

Même si je ne suis pas très vieux, mais c'est plaisant d'acheter du neuf, c'est valorisant, il y a tout cet attrait-là, mais après, là, aujourd'hui, je me dis « voilà, ce n'est plus mes intérêts", enfin, je n'ai plus envie de montrer quelque chose via ma voiture.

[...] Je pense [pour donner un exemple] que l'Astra, oui, la première voiture neuve que j'ai achetée, oui, parce qu'il a fallu que j'achète toutes options, c'était... c'était ma première voiture neuve que j'achetais avec mon métier de conducteur, vous voyez (Ludovic)

D'un objet indispensable pour se déplacer et se positionner dans un espace indissociablement social et géographique dont l'absence témoignerait d'une forme d'exclusion, la voiture devient, dans une réalité toute différente (la région parisienne), un objet coûteux, stressant et qui ne permet plus à son propriétaire de rendre compte de son positionnement (Christophe) ou de son ascension sociale (Ludovic). Pour le dire autrement, les ressorts de la définition et de la perception de la misère automobile sont variables d'un individu à l'autre mais peuvent l'être aussi tout au long de leurs trajectoires de vie. Si la socialisation primaire et ses instances (famille, école, groupe d'appartenance) jouent un rôle important dans la représentation initiale de l'espace des positions et des rapports à l'automobile, cette représentation et ses conséquences pratiques peuvent évoluer avec une socialisation secondaire, professionnelle, amicale mais aussi spatiale.

\section{Conclusion : l'espace des positions automobiles à travers l'expérience vécue}

Pour conclure, les expériences décrites dans cet article rendent compte de ce que nous avons appelé la misère de position automobile et de ses différentes formes. Les cas discutés n'épuisent pas l'ensemble des configurations possibles en la matière. Néanmoins, ils montrent comment l'équipement automobile est le signe d'une pauvreté 
(les pauvres se distinguent clairement par leur équipement automobile des riches) ou d'un état d'appauvrissement qui est surtout relatif. Si la misère de position automobile est mise en lumière par l'enquêteur comme par l'enquêté'e l'expérience de celle-ci est loin d'être homogène.

Recourir à la misère de position automobile permet aussi d'insister sur les multiples ressorts de l'expérience vécue de l'automobile, montrant que cet objet est susceptible de vulnérabiliser la situation économique des ménages de manière objective, mais aussi leur identité sociale. Pour comprendre ce double caractère du rapport à l'automobile, nous avons montré que les cultures automobiles ne peuvent être homogénéisées. Elles peuvent être expliquées par des rapports sociaux entre classes sociales. Comme tout domaine culturel, le rapport à l'automobile est pluriel et dépendant de rapports de force sociaux et politiques. Par-delà leur rhétorique écologique, les politiques d'incitation à la démotorisation mentionnées en introduction peuvent être lues comme des entreprises politiques mues par une logique de classe, c'est-à-dire porteuses d'un antagonisme entre des groupes sociaux et d'un traitement contradictoire des situations, pénalisant les uns et favorisant d'autres.

Dans cette perspective, le prisme de la misère de position automobile, complémentaire de celui de misère de condition examinée plus intensivement par la littérature scientifique, permet de décrire avec finesse les situations d'individus qui sont peu à l'aise financièrement pour anticiper sereinement l'avenir de leur mobilité, soumis à une forte hétéronomie pour définir leurs pratiques de déplacements et ainsi fragilisés par les aléas. Autrement dit, pour transposer au domaine de la mobilité automobile des propos de Pierre Bourdieu, la misère de position automobile parle de «ceux qui occupent une position inférieure et obscure à l'intérieur d'un univers prestigieux et privilégié, expérience d'autant plus douloureuse [...] que cet univers, auquel ils participent juste assez pour éprouver leur abaissement relatif, est situé plus haut dans l'espace global ». Il faut bien entendu retenir la distinction posée dès l'introduction entre des formes strictement économiques d'un rapport contraint à l'automobile (parmi les ménages aux bas revenus) de situations de difficultés induisant dans les représentations, parfois par anticipation, un sentiment de déclassement possible au prisme des usages ou de la possession automobile. Si l'on admet cela, on peut comprendre la seconde configuration et les conséquences pratiques qu'elle entraîne comme une situation vécue d'hétéronomie vis-à-vis d'une forme de culture automobile s'imposant par "le haut», venant disqualifier d'autres formes de rapport possible (approche en partie suggérée par Luc Boltanski (1975) dans un autre contexte de définition de cette culture automobile).

Pour revenir aux termes de notre recherche, la misère de position automobile fait finalement référence à un fait qui nécessite d'être restitué dans un environnement multidimensionnel dépassant la seule évocation d'un "contexte » favorable ou non à l'automobile. La misère de position automobile tend à souligner une contrainte relationnelle de nécessité (et non causale) indissociablement morale, symbolique et économique par rapport à la place qu'occupent les individus d'une société donnée visà-vis de ce mode de déplacement. L'idée de misère de position automobile et la configuration sociale dans laquelle elle existe permettent de préciser le sens d'une expression comme " la voiture, c'est obligatoire ", en la restituant dans la stratification sociale et dans l'histoire. Elle permet de soulever des questions portant sur le comment, le quand ou les ressorts de la perception des alternatives à l'automobile, pour les 
réinscrire dans un continuum d'inégalités de position interdépendantes face à l'automobile et à ses «cultures » symboliques, économiques et politiques.

Pour certains, comme les professions intermédiaires commerciales dont l'activité professionnelle est mobile par définition, il y a « obligation » d'utiliser sa voiture pour obtenir ou se maintenir dans l'emploi. Pour d'autres, la misère de position renvoie à l'appauvrissement vécu ou perçu qui est lié pour tout ou partie à la possession ou l'usage d'une voiture personnelle et à leur abandon: la place de parking devient difficile à payer, les longs voyages motorisés deviennent trop coûteux, les modèles de voiture achetés deviennent de moins en moins prestigieux, les embouteillages à l'entrée des agglomérations sont de plus en plus contraignants parce qu'il y a obligation de vivre de plus en plus loin du lieu de travail, etc. Dans ces configurations, la voiture devient le signal d'une position subalterne dans un espace de position qui ne se réduit pas au modèle de véhicule possédé, mais plutôt à l'aisance dans les déplacements quotidiens et l'autonomie sociale apportée par l'automobile. Se débarrasser de la voiture amène à des pratiques de mobilité jugées jusqu'alors comme pénibles et qui matérialisent la dépendance et la perte d'une autonomie, plus perçue que réelle, pour la mobilité quotidienne : marche, utilisation de transports en commun, changement d'activité et des lieux qui y sont associés.

Dans cette configuration, la misère de position automobile amène à fréquenter des personnes en misère de condition de mobilité. Prenons l'exemple d'un voyage en RER le matin où se côtoient des employées relativement bien rémunérée's, mais subalternes et des ouvrierère's. Toutes et tous partagent une condition liée à la mobilité où les coûts automobiles sont abordables pour une petite fraction de ménages. Ce partage de condition peut, en interaction, conduire un individu qui n'y avait jusqu'alors pas songé à se sentir «au bas de l'échelle». Mais il se pourrait aussi que la communauté de condition en termes de mobilité entraine, parfois, des solidarités ou des réévaluations de catégories de jugement ordinaire sur les autres, qui n'auraient pas eu lieu autrement.

\section{BIBLIOGRAPHIE}

Boltanski L. (1975), « Les usages sociaux de l'automobile : concurrence pour l'espace et accidents ", Actes de la recherche en sciences sociales, vol. 1, n², p. 21-49.

Bourdieu P. (dir.) (1993), La misère du monde, Paris, Seuil.

Brown A. E. (2017), « Car-less or Car-free? Socioeconomic and Mobility Differences Among Zerocar Households », Transport Policy, n 60, p. 152-159.

Cacciari J. et Belton Chevallier L. (2020), « La démotorisation des ménages comme analyseur de la diversité des expériences de socialisation à la "norme automobile" », Flux [en ligne], $n^{\circ} 119-120$

Cartier M., Coutant I., Masclet O. et Siblot Y. (2008), La France des « petits-moyens ». Enquête sur la banlieue pavillonnaire, Paris, La Découverte. 
Clark B., Chatterjee K. et Melia S. (2016), « Changes in Level of Household Car Ownership: The Role of Life Events and Spatial Context », Transportation, $n^{\circ} 43$, p. 565-599.

Collectif Rosa Bonheur (2019), La ville vue d'en bas. Travail et production de l'espace populaire, Paris, Éditions Amsterdam.

Coquart B. (2018), « L'investissement dans la voiture compense la délocalisation des emplois », Les jours [en ligne], $\mathrm{n}^{\circ} 7$, disponible sur http://lesjours.fr/obsessions/route/ep7-interview-benoitcoquard/, consulté le 12/12/2019.

Coulangeon P. et Petev I. (2012), « L'équipement automobile, entre contrainte et distinction sociale ", Économie et statistique, $\mathrm{n}^{\text {os }} 457-458$, p. 97-121.

Demoli Y. (2015), Automobile et stratification sociale : diffusion, caractéristiques et coûts de l'équipement automobile en France depuis les années 1980, Sciences Po Paris, thèse de doctorat en sociologie.

Demoli Y. (2017), « Les jeunes et la voiture, un désir contrarié ? ", Métropolitiques [en ligne], disponible sur https://www.metropolitiques.eu/Les-jeunes-et-la-voiture-un-desir-contrarie, consulté le 12/12/2019.

Demoli Y. et Lannoy P. (2019), Sociologie de l'automobile, Paris, La Découverte.

Espinasse C. (2005), « Le deuil de l'objet voiture chez les personnes âgées », rapport final pour le PREDIT.

Fol S. (2009), La mobilité des pauvres : pratiques d'habitants et politiques publiques, Paris, Belin.

Froud J., Johal S., Leaver A. et Williams K. (2002), « Not Enough Money: The Resources and Choices of the Motoring Poor », Competition and Change, vol. 6, $\mathrm{n}^{\circ}$ 1, p. 95-111.

Grimal R. (2012), « Des mobilités plus homogènes ou plus diversifiées ? ", Économie et statistique, $\mathrm{n}^{\text {os }} 457-458$, p. 13-34.

Grossetête M. (2012), Accidents de la route et inégalités sociales : les morts, les médias et l'État, Vulaines-sur-Seine, Éditions du Croquant.

Klein N. J. et Smart M. J. (2017), « Car Today, Gone Tomorrow: The Ephemeral Car in Lowincome, Immigrant and Minority Families ", Transportation, vol. 44, n 3, p. 495-510.

Le Breton É. (2005), Bouger pour s'en sortir : mobilité quotidienne et intégration sociale, Paris, Armand Colin.

Mattioli G. et Colleoni M. (2016), « Transport Disadvantage, Car Dependence and Urban Form », in Pucci P. et Colleoni M. (dir.), Understanding Mobilities for Designing Contemporary Cities, Springer International Publishing, p. 171-190.

Motte-Baumvol B. et Belton Chevallier L. (2019), « Une immobilité quotidienne marginale? Analyse critique des concepts et des pratiques ", in Bernier X. (dir.), Mobilités et Marginalités, Rennes, Presses Universitaires de Rennes, p. 43-58.

Nicolas J.-P., Vanco F. et Verry D. (2012), « Mobilité quotidienne et vulnérabilité des ménages », Revue d'économie régionale et urbaine, $\mathrm{n}^{\circ} 1, \mathrm{p} .19-44$.

Orfeuil J.-P. (2004), Transports, pauvretés, exclusions, Paris, Éditions de l'Aube, p. 27-47. 


\section{RÉSUMÉS}

De nombreuses politiques urbaines tentent aujourd'hui de réduire la place de l'automobile au cœur des villes françaises. Si elle peut se révéler variée, l'appréciation de cette incitation « par le haut » est souvent peu analysée du point de vue des ménages, notamment ceux issus des classes populaires, les plus à même d'être vulnérabilisés par l'abandon de la voiture. Dans cet article nous montrerons qu'au-delà de leurs conditions économiques absolues, les rapports des ménages à la voiture rendent compte d'une misère de position qui renvoie à la perception de leur positionnement dans l'espace social et qui est susceptible d'évoluer au gré de leur trajectoire, de leur socialisation. L'argumentation de l'article s'appuie sur une enquête par entretiens biographiques menée dans quatre aires urbaines françaises (Bordeaux, Dijon, Lyon et Paris) auprès de personnes ayant renoncé, par contrainte ou non, à l'usage ou à la possession de véhicule motorisé pour les déplacements quotidiens.

Several recent urban policies plan to reduce the role of the automobile in French cities. While it may be varied, households' appreciation of this "top-down" incentive is often poorly analysed, especially if it comes from working class households that are most likely to be weakened by the dropping out their car. Through this article, we will show that, beyond their absolute economic conditions, household relations with the car reflect a misery of position, which refers to the perception of their positioning in the social space and which is likely to change according to their trajectory, their socialization. The argumentation of the article is based on a biographical interview survey conducted in four French urban areas (Bordeaux, Dijon, Lyon and Paris) with people who have given up, by force or not, the use or possession of a motor vehicle for daily travel.

\section{INDEX}

Mots-clés : socialisation, misère de position, misère de condition, automobile, aires urbaines

Keywords : socialization, poverty of position, poverty of condition, car, urban areas

\section{AUTEURS}

\section{JOSEPH CACCIARI}

ATER

Université Paris Nanterre

\section{LESLIE BELTON CHEVALLIER}

Chargée de recherches

Université Gustave Eiffel 